\title{
The Effect of Plasma Profile Variation on the Stability of the $n=1$ Internal Kink Mode in Rotating Tokamak Plasmas
}

\author{
IT Chapman ${ }^{1}$, JP Graves ${ }^{2}$, C Wahlberg ${ }^{3}$ and the MAST Team \\ ${ }^{1}$ EURATOM/UKAEA Fusion Association, Culham Science Centre, Abingdon, \\ Oxfordshire OX14 3DB, United Kingdom \\ 2 École Polytechnique Fédérale de Lausanne (EPFL), Centre de Recherches en \\ Physique des Plasmas, Association EURATOM-Confédération Suisse, 1015 \\ Lausanne, Switzerland \\ ${ }^{3}$ Department of Physics and Astronomy, EURATOM/VR Fusion Association, PO \\ Box 516, Uppsala University, SE-751 20 Uppsala, Sweden \\ E-mail: ian.chapman@ukaea.org.uk
}

\begin{abstract}
.
The sensitivity of the stability of the ideal $n=1$ internal kink mode is analysed both analytically and numerically in rotating tokamak plasmas. These stability analyses have been carried out including the centrifugal effects of toroidal plasma rotation upon the equilibrium, and also inconsistently when the equilibrium is treated as static. The plasma stability is partially (consistent equilibrium) or wholly (inconsistent treatment) determined by the radial profiles of the plasma density and rotation velocity. It is found that the internal kink mode stability is strongly influenced by small variations in these plasma profiles. Indeed, modest perturbations to the profiles inside the $q=1$ surface of only a few percent can result in a stabilising effect upon the kink mode with respect to the static mode growth rate becoming a destabilising effect at the same rotation amplitude, or vice versa. The implications of this extreme sensitivity are discussed, with particular reference to experimental data from MAST.
\end{abstract}




\section{Introduction}

Modern-day tokamaks heated by neutral beam injection (NBI) can rotate toroidally at a significant fraction of the Alfvén speed [1-4]. It has been shown that such strong toroidal rotation can ameliorate or suppress many magnetohydrodynamic (MHD) instabilities, resulting in significantly improved plasma performance. For instance, resistive wall modes can be stabilised by sufficiently fast toroidal rotation [4-9]; ballooning modes are stabilised by sheared toroidal flows [10-14] leading to a change in edge localised mode behaviour [14-18]; the internal kink mode is stabilised by rotation [19,20] resulting in a modification of the sawtooth behaviour [21-23] and quasi-interchange modes [24,25] and infernal modes [26] can also be stabilised by plasma flows. Thus it is of considerable interest, firstly, to understand how toroidal rotation affects MHD instabilities and, secondly, to be able to model this stabilisation in order to optimise plasma operational scenarios and make predictions for ITER and other next-step devices.

In current tokamaks, the strong toroidal plasma rotation is usually provided by the external momentum input from neutral beam injection [3]. However, due to a combination of the large plasma volume with respect to NBI power, the high plasma density and the limitations of beam current, it is anticipated that ITER [27] and future fusion reactors will rotate at a much slower speed. Thus, it is of critical importance to assess whether the toroidal rotation in current devices plays a key role in MHD stability, which may then be absent in ITER. The role of rotation is also of paramount importance in spherical tokamaks (STs), where toroidal flows can be especially large due to the small plasma volume per unit of NBI power and low moment of inertia of the plasma. Indeed, it is understood that high- $\beta$ operation of NSTX is achieved partially through the rotational stabilisation of MHD instabilities [26,28]. Furthermore, the design of future STs, such as the Component Test Facility (ST-CTF) [29-31] are predicated upon the stabilising effects of strong toroidal rotation. Consequently, it is vital to assess not only the impact of toroidal flow on MHD stability, but also the plasma parameters under which (de)stabilisation occurs.

In recent years many MHD stability codes have been extended to include the effects of rotation in order to make an assessment of the stabilising role of toroidal flows. MARSF [32], Elite [33], Mishka-F [34] and Minerva [35], amongst others, have been used to study the effect of rotation on the stability of RWMs [32,36], ELMs [15,33] and sawteeth $[34,37,38]$. In all of these codes, the flow is not included self-consistently in the equilibrium, but only perturbatively in the stability analyses. However, there are some stability codes that do treat the equilibrium flow self-consistently: FLOS [40] which uses a flowing equilibrium from the FLOw code [41], the PHOEnIX code [42] which couples to the equilibrium code Finesse [43], the CASTOR-Flow code [44] which uses an equilibrium derived from the Diva code [45] and the M3D code [37].

In reference [39] we discuss the applicability of using a non-consistent treatment of the plasma rotation only in the stability analysis and not in the equilibrium formulation. Whilst this can be a valid approximation, particularly at very sub-Alfvénic rotation 
velocities, it can also lead to significantly misleading, or even erroneous results. In this paper we concentrate on the sensitivity of profile variation in determining MHD stability in a rotating plasma. In section 2 the effect of plasma flow on the $n=1$ internal kink mode is assessed for different equilibrium profiles with both a consistent and inconsistent treatment. Section 3 shows that small changes in the equilibrium profiles can result in a very significant change in the stability of the kink mode in flowing plasmas. This could be of great significance for the optimisation of plasma operational scenarios to enhance rotational stabilisation. The stability of the internal kink in MAST plasmas is analysed in section 4 and the sensitivity of the results to perturbations in the equilibrium profiles is assessed. Finally, the implications of these results are discussed in section 5 .

\section{Effect of Equilibrium Profiles on Kink Mode Stability Using Consistent and Inconsistent Treatments}

The stability of the $n=1$ internal kink mode can be significantly influenced by toroidal plasma rotation [19-21,39]. It is clear from reference [39] that the profiles of the plasma density and rotation play a strong role in determining the mode stability. In order to investigate how variation in plasma profiles affects MHD stability we compare predictions from a large aspect-ratio analytical theory with full toroidal numerical analyses. The CASTOR-FLOW code [44], which includes the centrifugal effects on the plasma equilibrium, and the MisHKA-F code [34], which treats the equilibrium flow only in the perturbation analysis, have both been employed to study how plasma flow affects the $n=1$ kink mode. Since both codes have been derived from the CASTOR ideal MHD stability code [46,47], any differences observed are resultant from the treatment of the equilibrium flow rather than from the numerical formulation of the eigenvalue solvers.

The sensitivity of the rotational stabilisation to variations in the plasma profiles has also been analysed using a large aspect ratio expansion of the compressible magnetohydrodynamic equations including equilibrium flows. The pressure, and also the density, profiles are taken such that

$$
\begin{aligned}
& \frac{p(r, \theta)}{p_{0}(r)}=\frac{\rho(r, \theta)}{\rho_{0}(r)}=\exp \left[\frac{\sigma_{1} \rho \Omega^{2}\left(R^{2}-R_{0}^{2}\right)}{2 p}\right] \\
& \beta_{p}=-\frac{2 \mu_{0} R_{0}^{2} q^{2}}{B_{0}^{2} r^{4}} \int_{0}^{r} r^{\prime 2} \frac{\mathrm{d}}{\mathrm{d} r}\left(p_{0}+\sigma_{2} \mathcal{M}^{2} p_{0}\right) \mathrm{d} r^{\prime}
\end{aligned}
$$

where $p, \rho, \Omega$ are the plasma pressure, density and rotation frequency respectively, $\sigma_{1}, \sigma_{2}=0,1, R$ and $r$ are the plasma major and minor radius respectively, $B_{0}$ is the toroidal field at the magnetic axis and $\mathcal{M}$ is the sonic Mach number, $\mathcal{M}=\sqrt{\rho \Omega^{2} R_{0}^{2} / 2 p_{0}}$. The equilibrium plasma flow is included self-consistently when $\sigma_{1}=\sigma_{2}=1$. When $\sigma_{1}=0$, the tangential gradients of the density and pressure on the flux surfaces are neglected, whereas $\sigma_{2}=0$ neglects the ancillary Shafranov shift induced by the centrifugal force. Following the detailed analysis given in reference [39], the growth rate of the $n=1$ internal kink mode in flowing plasmas is given by

$$
\gamma=\sqrt{\gamma_{0}^{2}-\omega_{G A M}^{2}}
$$


where

$$
\frac{\gamma_{0}}{\omega_{A}(0)}=-\frac{\pi \delta \hat{W}}{\sqrt{3} s_{1}}\left(\frac{\rho_{0}(0)}{\rho_{0}\left(r_{1}\right)}\right)^{1 / 2}\left(\frac{r_{1}}{R_{0}}\right)^{2}
$$

and

$$
\omega_{G A M}^{2}=\frac{\sigma_{1} \mathcal{M}^{2} \Omega^{2}}{3}\left(1-\frac{1}{\Gamma}\right)+\cdots
$$

$\Gamma$ is the adiabatic index, $\omega_{A}$ is the Alfvénic frequency $\omega_{A}^{2}=B_{0}^{2} / \mu_{0} \rho_{0} R_{0}^{2}, s_{1}$ is the magnetic shear at the $q=1$ surface, $s=r / q \mathrm{~d} q / \mathrm{d} r$ and

$$
\delta \hat{W}=\delta \hat{W}_{\text {Bussac }}+\delta \hat{W}_{\text {Rot } 1}+\delta \hat{W}_{\text {Rot } 2}
$$

The fluid drive, as calculated in reference [48], has been extended in accordance with reference [49] as

$$
\delta \hat{W}_{\text {Bussac }}=\Delta q\left[\frac{13}{48}-3 \beta_{p}^{2}\right]+(\Delta q)^{2}\left[\frac{5}{32}-\frac{5 \beta_{p}}{2}+6[1+\ln (\Delta q)] \beta_{p}^{2}\right]+\cdots(7)
$$

whilst the contributions arising in the presence of toroidal rotation are [39]

$$
\begin{aligned}
& \delta \hat{W}_{\text {Rot } 1}=\frac{\hat{\Omega}^{2}}{2}\left(\frac{2}{\epsilon_{\Omega}^{2}}-\frac{1}{\epsilon_{\rho}^{2}}\right) \\
& \delta \hat{W}_{R o t 2}=\left(\sigma_{2}-1\right) \beta_{p} \hat{\Omega}^{2}\left(\frac{2}{\epsilon_{\Omega}^{2}}+\frac{1}{\epsilon_{\rho}^{2}}\right)
\end{aligned}
$$

where $\hat{\Omega}=\Omega(0) / \omega_{A}(0)=V_{0} / V_{A}$, the density and rotation profiles are assumed to be parabolic,

$$
\begin{aligned}
& \Omega(r)=\Omega(0)\left[1-\frac{r^{2}}{\epsilon_{\Omega}^{2} R_{0}^{2}}\right. \\
& \rho(r)=\rho(0)\left[1-\frac{r^{2}}{\epsilon_{\rho}^{2} R_{0}^{2}}\right.
\end{aligned}
$$

and the profile length scales are defined as $\epsilon_{\rho}=r_{\rho} / R_{0}$ and $\epsilon_{\Omega}=r_{\Omega} / R_{0}$. Equation 7 is strictly only valid in the limit of small $\Delta q$. It is evident from the presence of the $\epsilon_{\rho}$ and $\epsilon_{\omega}$ terms in equations 8 and 9 that the details of the density and rotation profile can influence the stability of the internal kink mode in flowing plasmas.

When the centrifugal effects on the equilibrium are neglected, which is to say that $\sigma_{1}=0$ in equation 1 and $\sigma_{2}=0$ in equation 9 , the dominant effect of rotation on the stability of the plasma arises from the radial profiles of the plasma density and rotation. When the flow effects are included in the equilibrium, the profile effects remain, but an additional stabilisation appears due to the coupling of the kink mode with the lowfrequency geodesic acoustic mode (GAM) [50]. This mechanism depends solely on the rotation at the $q=1$ radius. Reference [39] analyses the applicability of the inconsistent treatment of the equilibrium flow and the conditions under which the agreement with the consistent treatment is satisfactory. Here, we are interested in how the plasma profiles affect the kink mode stability in both the consistent and inconsistent treatments. 
In order to investigate this effect we have employed a simple model equilibrium with a pressure profile given by

$$
p(r)=p(0)\left(1-\frac{r^{2}}{r_{p}^{2}}\right)
$$

where $r_{p}$ is a constant. The plasma has a circular boundary, an inverse aspect ratio $\epsilon_{a}=0.1$, poloidal beta $\beta_{p}=0.3$ and central beta $\beta_{0}=2 \mu_{0} p_{0} / B_{0}^{2}=0.66 \%$. The safety factor on-axis is $q_{0}=0.938$, and $r_{1}=0.3 a$. The equilibrium is unstable to the ideal $n=1$ internal kink mode. The stability of this equilibrium to the internal kink mode is tested with respect to toroidal rotation for four different combinations of density and flow profiles, such that

$$
\begin{aligned}
& \rho(r)= \begin{cases}\rho(0) & \text { Flat Density } \\
\rho(0)\left(1-\frac{r^{2}}{r_{\rho}^{2}}\right) & \text { Parabolic Density }\end{cases} \\
& \Omega(r)= \begin{cases}\Omega(0) & \text { Flat Rotation } \\
\Omega(0)\left(1-\frac{r^{2}}{r_{\Omega}^{2}}\right) & \text { Parabolic Rotation }\end{cases}
\end{aligned}
$$

For each of these combinations of profiles, together with a parabolic pressure profile, the $n=1$ mode growth rate has been found analytically according to equation 4 and numerically using both MishKA-F and CASTOR-FlOW. The inconsistent treatment employs a static equilibrium generated by the fixed boundary HELENA code [51], whereas the consistent treatment with the CAstor-Flow code employs the freeboundary equilibrium code DIVA [45] to generate an equilibrium that includes the strong toroidal rotation. By increasing the toroidal rotation systematically in both the equilibrium calculation and the perturbation analysis, the toroidal flow required to marginally stabilise the $n=1$ internal kink mode can be calculated and compared to the result from the non-self-consistent MisHKA-F analysis. The growth rate arising from the two calculations in the static case differs marginally because of the difference between the free- and fixed-boundary equilibria. The Helena code is constrained to the circular plasma shape, whereas the Diva code takes as input the currents in the poloidal field coils and calculates the resultant plasma configuration. Consequently, there is an imperceptible difference in the plasma shape which results in a very small difference in the static growth rate. Care was taken to match the static $q$-profiles, in particular the radial location of the $q=1$ surface, arising from the two equilibrium codes.

Figure 1 shows the growth rate of the $n=1$ kink mode with respect to the toroidal flow at the magnetic axis for each of the four combinations of plasma profiles when the equilibrium flow is treated self-consistently. It is clear that variation in the profiles has a significant effect on the mode stability, due to a strong effect from GAM coupling [39]. When the plasma rotation is flat and the density is parabolic the toroidal flow required for marginal stability increases by $\sim 30 \%$ compared with a plasma with flat density and parabolic flow. Since it is the gradient of the profiles in the region of $q=1$ that is important, the concept of both flat and parabolic profiles is highly plausible. Thus, it is 
evident that the rotational stabilisation of the mode is reliant upon the precise plasma profiles. This sensitivity to the profiles is substantially increased if an inconsistent treatment of the equilibrium flow is employed. Figure 2 again shows the growth rate of the kink mode as a function of the toroidal rotation on axis for the four combinations of plasma profiles when the equilibrium rotation is only included inconsistently in the stability analysis. It is clear that the profiles play a key role in determining kink mode stability when an inconsistent flow analysis is performed. For instance, assuming a parabolic flow profile, then changing the density profile from flat to parabolic results in a rotationally-stabilised mode (at $V_{0}=0.01 V_{A}$ ) becoming strongly unstable. This situation could be envisaged in tokamak plasmas as the density profile peaks during the discharge evolution $[52,53]$. Whilst this inconsistent analysis is clearly not valid for certain plasma profiles, particularly at large toroidal rotation velocities [39], such approximations are still employed in many numerical studies [32-35]. When toroidal flow is neglected from the equilibrium it is particularly important to consider variations in the plasma profiles, since the stability of the kink mode can be highly sensitive to such perturbations. The final thing to note is the excellent agreement between the numerical results and the analytical predictions from equation 4 . Whilst such accordance is not anticipated at smaller aspect-ratio, the qualitative behaviour is expected to agree.

In order to test this, the effect of profile variation on the stability of the $n=1 \mathrm{kink}$ mode has also been tested for a realistic tokamak aspect ratio. Once again, a simple equilibrium with a pressure profile given by equation 12 is employed. The plasma has a circular boundary, an inverse aspect ratio $\epsilon_{a}=0.3$, poloidal beta $\beta_{p}=0.3$ and central beta $\beta_{0}=2 \mu_{0} p_{0} / B_{0}^{2}=5.6 \%$. The safety factor on-axis remains as $q_{0}=0.938$, and $r_{1}=0.3 a$. The growth rate of the mode with respect to toroidal rotation is illustrated in figures 3 and 4 for the consistent and inconsistent treatment respectively. Again, it is clear that the stability of the kink mode is very dependent upon the plasma profiles, particularly in the inconsistent case. Whilst there is not perfect agreement between the numerical results using full toroidal geometry and the analytical predictions using large aspect-ratio approximations, the qualitative behaviour agrees well. When the equilibrium flow is treated consistently, variation in the profiles at the $q=1$ surface such that an equilibrium with parabolic flow and flat density is perturbed to have flat rotation and parabolic density results in the toroidal rotation required to stabilise the $n=1$ mode increasing by more than a factor of two. Similarly, if the equilibrium flow is treated inconsistently, the same change in profiles results in a strongly stabilised mode being driven very unstable.

It is also of interest to consider the effect of profile variation upon the stability of the kink mode in a plasma with a lower central safety-factor, as often observed experimentally in tokamaks. Figure 5 shows the growth rate of the mode as a function of rotation for a circular plasma, with an inverse aspect ratio $\epsilon_{a}=0.3, q_{0}=0.75$, poloidal beta $\beta_{p}=0.3$ and central beta $\beta_{0}=2 \mu_{0} p_{0} / B_{0}^{2}=5.6 \%$. Again, there is qualitative agreement between the analytic curves and the numerical computation, though the quantitative comparison is degraded at high $\beta_{p}$ since the analytic approximations 
become less rigorous. Nonetheless, it should be noted that in both theory and simulation, there exists a regime where increasing the sheared rotation - present in the parabolic flow curves - drives the mode more unstable initially. This is in contrast to figure 3 where the parabolic flow was always stabilising and the parabolic density-flat flow profiles resulted in the most unstable mode. With a larger $\Delta q$, the parabolic density-parabolic flow case becomes the most unstable profile combination. This happens since at $\Delta q=0.25$, the increase in sheared flow leads to a more substantial increase in the poloidal beta and enhanced Shafranov shift. This in turn results in an increase in $\delta W_{\text {Bussac }}$ as seen in equation 7 , which increases the growth rate of the mode. At sufficiently high rotation, the absolute rotation contained in the inertia terms in equations 8 and 9 begins to dominate and the mode is stabilised. This result emphasises how subtle changes in the equilibrium profiles can lead to different behaviour in the stability of the $n=1$ mode due to the competition between the destabilising enhanced Shafranov shift and the stabilising inertia term.

Finally, it is also evident that the sensitivity of the stability of the $n=1$ kink mode to variation in the plasma profiles is heightened as the inverse aspect ratio increases [39]. At very large aspect ratio, the profile sensitivity is weak since the geodesic acoustic mode stabilisation term, given by equation 5 , dominates over the $\delta \hat{W}_{\text {Rot } 1}$ term from equation 8 in the mode growth rate (equation 3). The GAM stabilisation does not depend upon the density and flow profiles, only the rotation at the $q=1$ surface. This increased sensitivity with increasing $\epsilon$ is evident by the wider range in the rotation at marginal stability for the four profile combinations in figure 3 , where $\epsilon_{a}=0.3$, than in figure 1 , where $\epsilon_{a}=0.1$.

It is clearly evident from the behaviour exhibited in figures 1-5 that MHD stability in flowing tokamak plasmas is highly sensitive to the plasma profiles. This sensitivity is examined in more detail in section 3.

\section{Sensitivity of Kink Mode Stability to Perturbations in Plasma Profiles}

The comparison between consistent and inconsistent flow treatments in section 2 shows that the variation in the rotation and density profiles significantly affects the stabilising role of plasma flow. In order to investigate the sensitivity of the mode stability to these profiles, we analytically calculate the growth rate for a range of different parabolic profiles. The density profile is defined by

$$
\frac{\rho(r)}{\rho(0)}=1-\frac{r^{2}}{r_{\rho}^{2}}=1-\left(\frac{\epsilon_{a}}{\epsilon_{\rho}}\right)^{2}\left[\frac{r}{a}\right]^{2}
$$

and the rotation profile is defined as

$$
\frac{\Omega(r)}{\Omega(0)}=1-\frac{r^{2}}{r_{\Omega}^{2}}=1-\left(\frac{\epsilon_{a}}{\epsilon_{\Omega}}\right)^{2}\left[\frac{r}{a}\right]^{2}
$$

We begin from the case with $\epsilon_{a}=0.3$, a flat rotation profile $\left(\epsilon_{\Omega}=\infty\right)$ and a parabolic density profile $\left(\epsilon_{\rho}=0.3\right)$ which results in the most unstable plasma, as seen in figure 3 . 


\begin{tabular}{cccc}
\hline$\epsilon_{\rho}$ & $\left(\epsilon_{a} / \epsilon_{\rho}\right)^{2}$ & $\rho\left(r_{1}\right) / \rho(0)$ & Change in $\rho\left(r_{1}\right)$ \\
\hline 0.3 & 1.0 & 0.91 & - \\
0.28 & 1.148 & 0.897 & $-1.5 \%$ \\
0.26 & 1.331 & 0.880 & $-3.3 \%$ \\
0.24 & 1.563 & 0.859 & $-5.6 \%$ \\
0.22 & 1.860 & 0.833 & $-8.5 \%$ \\
\hline
\end{tabular}

Table 1. Table showing different density profiles for a circular plasma with $\epsilon_{a}=0.3$, $\epsilon_{\Omega}=\infty$ and $r_{1}=0.3 a$.

\begin{tabular}{cccc}
\hline$\epsilon_{\Omega}$ & $\left(\epsilon_{a} / \epsilon_{\Omega}\right)^{2}$ & $\Omega\left(r_{1}\right) / \Omega(0)$ & Change in $\Omega\left(r_{1}\right)$ \\
\hline$\infty$ & 0 & 1 & - \\
$0.6 i$ & -0.25 & 1.023 & $+2.3 \%$ \\
$0.5 i$ & -0.36 & 1.032 & $+3.2 \%$ \\
$0.4 i$ & -0.56 & 1.051 & $+5.1 \%$ \\
$0.3 i$ & -1.0 & 1.09 & $+9.0 \%$ \\
\hline
\end{tabular}

Table 2. Table showing different flow profiles for a circular plasma with $\epsilon_{a}=0.3$, $\epsilon_{\rho}=0.3$ and $r_{1}=0.3 a$.

The density and rotation profile parameters are varied such that the normalised density and rotation at the $q=1$ surface vary by less than $10 \%$, as outlined in tables 1 and 2 .

The resultant density and rotation profiles are shown in figures 6 and 7 respectively. The unphysical inverted rotation profiles are chosen to demonstrate the competition between the gradients in the density and flow profiles, as delineated by equation 8 where $\delta \hat{W}_{\text {Rot } 1} \propto 2 / \epsilon_{\Omega}^{2}-1 / \epsilon_{\rho}^{2}$. The growth rate of the $n=1$ internal kink mode as a function of toroidal velocity for each of the density profiles illustrated in figure 6 is shown in figure 8. It is clear that a very small change in the density profile can have a very significant affect on the stability of the internal kink mode. Indeed, a change in the gradient of the density at $q=1$ of only a few percent can result in destabilisation of the mode instead of stabilisation at a given toroidal rotation, or vice versa. A similar sensitivity is exhibited with respect to the rotation profile. Figure 9 shows the growth rate of the $n=1$ mode as a function of the toroidal rotation at the magnetic axis for the different flow profiles illustrated in figure 7 . Again, it is clear that a very small change in the plasma rotation profile can result in a substantial change in the mode stability. Once more, a change in rotation of the order of one percent can result in a rotationallystabilised mode becoming strongly unstable. Naturally, there is approximately double the effect if both rotation and density profiles vary simultaneously.

This result has significant implications for our understanding of the effect of rotation on the $n=1$ internal kink mode in tokamak plasmas. Whilst there is a predilection towards the assumption that sheared toroidal flows will stabilise the mode, these results show that this is highly sensitive to the exact rotation and density profiles. 
Furthermore, under certain conditions, the toroidal rotation can result in an enhanced destabilisation of the mode. Another consequence of these findings is an increase in the uncertainty of modelling stability in experimental flowing plasmas. A small error bar in the measurement of the rotation profile or density profile can result in misleading stability predictions. For instance, for a given rotation amplitude (in this case $v_{0}=0.03 v_{A}$ ), the rotation leads to an increase in mode stability relative to the static case for $\epsilon_{\rho}=0.28$, whereas the flow destabilises the mode relative to the static case when $\epsilon_{\rho}=0.3$. To this extent, figures 8 and 9 indicate that an uncertainty of only $\sim 1 \%$ can differentiate between stabilisation or destabilisation relative to the static growth rate, whereas typically the error associated with charge exchange measurements of the toroidal rotation and Thomson scattering measurements of the electron density can be $\sim 10 \%$ or larger. Consequently, when performing stability analyses for experimental plasmas it is important to consider the ramification of variations in the plasma profiles for kink mode stability.

Of course, there are limitations to using a large aspect-ratio approximation. However, the excellent agreement between the analytic theory and full toroidal geometry numerical simulations shown in section 2 suggests that the salient features of these analytical predictions will be present in numerical simulations too. Small variations in real tokamak plasma profiles are investigated numerically in section 4 .

\section{Kink Mode Stability in MAST plasmas}

The toroidal velocity in spherical tokamaks such as MAST can be very large, regularly approaching the ion sound speed, $\mathcal{M}=1$. Previous studies concluded that at typical MAST rotation speeds, the $n=1$ internal kink mode is strongly stabilised $[21,54]$. The linear MHD stability analyses in references [21,54] employ an inconsistent treatment of the equilibrium flow. The results in section 2 suggest that this may lead to substantial inaccuracies for certain plasma profiles. In order to assess whether the previous conclusion - that is to say that the sawtooth behaviour in MAST can be explained by the toroidal rotation arising from neutral beam injection - is valid, we have analysed the stability of the $n=1$ kink mode for the NBI-heated discharges discussed in reference [21]. For example, MAST discharge 13035 is heated with co-injected neutral beams of $1.82 \mathrm{MW}$ and has $I_{p}=730 \mathrm{kA}, B_{T}=0.408 \mathrm{~T}, \bar{n}_{e}=2.8 \times 10^{19} \mathrm{~m}^{-3}$ the safety factor at the magnetic axis predicted by the EFIT code [55] is $q_{0}=0.81$ and the maximum rotation speed was $v_{\phi} \approx 230 \mathrm{~km} / \mathrm{s}$, corresponding to $v_{0} / v_{A}=0.19$. The equilibrium is reconstructed by using the EFIT prediction for the current density profile and the pressure profile derived from the Thomson Scattering diagnostic, which provides profiles for the electron density and temperature. These profiles can be found in reference [21]. The soft X-ray diagnostic has been used to find the inversion radius of the sawtooth oscillations, and hence ascertain an estimate for the location of the $q=1$ surface. The pressure and current profiles, together with the fixed boundary shape of the last closed flux surface, are supplied to the HelenA code [51], which produces the 
equilibrium employed by MishKA-F [34]. The free-boundary equilibrium generated by the DIVA code [45] is generated by supplying the positions and currents of the poloidal field coils together with the plasma $\beta$ and position of the magnetic axis.

Figure 10 shows the growth rate of the $n=1$ internal kink mode with respect to the toroidal plasma rotation for MAST shot 13035 when both the inconsistent treatment with MishKA-F and the consistent CASTOR-Flow analysis have been employed. It is evident that for the rotation and density profiles in this discharge, and the tight aspect-ratio of the spherical tokamak, the difference in the point of marginal stability is $\sim 10 \%$. Stability analysis of other MAST sawtoothing discharges reveals that this is often the case, and indeed, in all the shots analysed in reference [21], the point of marginal stability never differs by more than 20\%. Furthermore, in all cases, the inconsistent treatment overestimates the toroidal rotation required to fully stabilise the mode, so the stability boundaries in previous studies provide an upper bound for the effect of rotation. Consequently, the conclusion that the different sawtooth behaviour exhibited in similar co- and counter-NBI heated MAST plasmas could be attributed to the direction and profile of the toroidal velocity is valid.

However, as discussed in section 3 , the stability of the $n=1 \mathrm{kink}$ mode, and particularly the difference arising between consistent and inconsistent treatment of the equilibrium flow, is eminently sensitive to the plasma profiles. As such, there are many MAST discharges where predictions for the point of marginal stability are highly dependent upon correct treatment of the equilibrium rotation. For instance, stability analyses have been performed for shot 18376, an H-mode sawtoothing discharge, quite typical of connected double null MAST plasmas. MAST discharge 18376 has $I_{p}=750 \mathrm{kA}, B_{T}=0.4 \mathrm{~T}, \bar{n}_{e}=1.93 \times 10^{19} \mathrm{~m}^{-3}$ and $q_{0}=0.9$. The density profile measured by the Thomson scattering diagnostic and the rotation profile found by charge exchange recombination spectroscopy are illustrated in figures 11 and 12 respectively. As before, the stability of the $n=1$ internal kink mode in the presence of strong toroidal rotation is modelled consistently using the CASTOR-FLOW code and inconsistently using MishKA-F. Figure 13 shows the mode growth rate as a function of toroidal velocity for both equilibrium flow treatments. It is evident that the rotation required to stabilise the mode predicted by inconsistent numerical analysis is vastly greater than that predicted by a code which includes the toroidal flow in the equilibrium formulation, at least for the plasma profiles in this MAST discharge. It is thus important to assess the mode linear stability with profiles that are as accurate as possible, or at least to consider how the error in profile diagnosis may affect the stability calculations. In general (at least for small $\Delta q$ ), a decreasing plasma density with minor radius is destabilising, whereas a decreasing velocity with minor radius is stabilising. The opposite is true for profiles that increase with minor radius. However, the nuances of the profiles and particularly their relative gradients mean that this rule of thumb may be misleading and accentuates the need for a full numerical analysis. That said, with a consistent treatment, the stabilising effect of coupling to the GAM induced by toroidal rotation will always dominate over other drives at sufficiently high rotation since equation 9 has a term proportional to $\Omega^{4}$. 
Finally, it is of interest to consider whether typical variations in the density and rotation profiles can lead to a substantial change in kink mode stability in MAST plasmas. The plasma profiles in MAST evolve significantly over the course of the discharge such that the kink mode stability may be substantially altered between different time points. In many co-NBI heated H-mode discharges in MAST, edge density 'ears' form as the edge transport barrier appears in the plasma. Over a short timescale (typically less than 100ms) the hollow density profile becomes peaked in the core [56,57]. Figure 14 shows the electron density profile as measured by the Thomson scattering diagnostic in MAST discharge 21430. The $q=1$ surface is near $\psi_{N}=0.4$, though it is moving outwards during the same time frame as the density profile evolution. Shot 21430 is a sawtoothing H-mode plasma in lower single null configuration heated with $2.79 \mathrm{MW}$ of NBI power, $I_{p}=700 \mathrm{kA}, B_{T}=0.4 \mathrm{~T}$ and $q_{0}=0.95$. As the plasma evolves the gradient of the density at the $q=1$ surface changes from positive to negative on a very short timescale. Over the same timescale, the toroidal rotation profile does not change significantly. Such temporal evolution of the density profile results in significant changes in the stability of the internal kink mode. Figure 15 shows the growth rate of the $n=1$ kink mode as a function of toroidal rotation for each of the density profiles illustrated in figure 14, with the flow profile assumed to be given by equation 16 . Soon after the transition to H-mode, when the density ears are formed, the kink mode is most readily stabilised by toroidal rotation. As the hollow density profile becomes more peaked, the kink mode becomes more unstable, and the stabilising effect of the toroidal flow is diminished. This is demonstrated by a doubling of the rotation velocity required to marginally stabilise the mode. Whilst the static MHD drive for the $n=1$ kink instability is also increased by the peaking of the density profile, the main reason for this marked increase in the marginality threshold is the reduced rotational stabilisation for these peaked density profiles.

The rotation profile can also vary substantially between MAST discharges when the NBI heating is oriented differently. The large distance between the vacuum vessel and the plasma in MAST means that the vertical position of the plasma can be varied by approximately half the minor radius. Consequently, the deposition position of the NBI can be varied by moving the plasma up and down significantly. In order to simulate the effect of moving the NBI deposition location further off-axis in MAST, the plasma is displaced vertically, such that beam deposition moves to well outside the $q=1$ surface [54]. The plasma current can also be reversed such that the toroidal current is oriented in the opposite direction to the direction of neutral beam injection [21]. Typical rotation profiles in each of these circumstances, together with double null discharges in H- and L-mode, are shown in figure 16. The profiles are normalised to the velocity at the magnetic axis. The amplitude of the rotation velocity is usually larger in counter-NBI heated plasmas than co-NBI shots, as counter-NBI results in large firstorbit losses caused by the fact that the injected ions move radially outward from the point of ionisation. A typical counter-injected trapped ion is lost on the outboard leg of its banana orbit, when it is moving in the co-current direction, resulting in enhanced 
angular momentum transfer to the plasma through the $J \times B$ torque [3]. The off-axis NBI heated shots usually rotate at slower rotation velocities than typical double-null connected discharges due to the increased neutral beam shine through when the beam is injected into lower density plasma further from the core. In order to consider how these different flow profiles affect kink mode stability in generic MAST plasmas, we have performed stability analyses with consistent treatment of the equilibrium flow for MAST discharge 20643 (an H-mode double null discharge). The pressure, current and density profiles are taken from the EFIT and Thomson scattering data, as described earlier, together with each of the plasma rotation profiles illustrated in figure 16. The density profile is approximately parabolic for this equilibrium reconstruction and does not exhibit the 'ears' shown in figure 13. The radial location of the $q=1$ surface for the static equilibrium is indicated in figure 16 by the tick mark on the $x$-axis and $\Delta q=0.12$. The equilibrium profiles and the different rotation profiles are, of course, not self-consistent with respect to one another. However, this approach is not intended to provide accurate stability boundaries, but only to demonstrate the phenomenological effect of variation in the rotation profile. The growth rate of the $n=1$ kink mode for each of these flow profiles is shown in figure 17. The rotation is measured in 17 in terms of $v(q=1) / v_{A}$ in order to demonstrate the effect of the rotation profile gradients at $q=1$ independent of the amplitude of the rotation at the rational surface. With the broader counter-NBI rotation profile, the rotation is less stabilising and the flow required to marginally stabilise the mode is increased. The most peaked double-null configuration rotation profile has the most stabilising effect on the $n=1$ mode. In practice, the enhanced spin-up experienced in counter-NBI plasmas is likely to compensate the less favourable rotation profile, resulting in broadly similar rotational stabilisation with both NBI orientations in MAST. It should be noted that an inconsistent treatment of the equilibrium flow would suggest that variation in the rotation profile may not change the rotational stabilisation as significantly, at least in the case of a peaked density profile [21].

Despite the sensitivity of the stability of the internal kink mode to variations in the equilibrium profiles, it should be noted that at sufficiently high toroidal rotation the stabilising GAM effects will always dominate in these MAST plasmas. Consequently, the empirical observation that rotation helps to stabilise the internal kink mode, manifest as sawteeth in tokamak plasmas, is well founded. However, the effect of the rotation profile is potentially of significance in experiments heated with balanced neutral beam injection. For instance, the DIII-D tokamak has one neutral beam injector permanently oriented opposite to the plasma current in addition to three co-current beams [58]. This can give rise to approximately null flow speed across the entire plasma radius but in the presence of flow shears, such that parts of the plasma are co-rotating, and parts are counterrotating [59]. Such exotic rotation profiles may give rise to rotational destabilisation of the internal kink mode when the flow profile has a positive gradient at the $q=1$ surface but the GAM damping term in equation 9 are negligible. However, in the case of DIII-D the fast ions arising from neutral beam injection are likely to dominate the stability of 
the $n=1$ kink mode, as discussed further in section 5 .

\section{Discussion and Conclusions}

The fast toroidal plasma rotation achieved in modern-day tokamaks predominantly arises from neutral beam injection heating. When neutral beams are injected tangentially into the plasma, the momentum of the beam particles is deposited in the plasma causing it to rotate. However, as well as imparting momentum to the bulk plasma, neutral beam injection also results in a population of energetic particles. It has been found that the introduction of a high-energy collisionless population of minority ions can strongly influence the stability of the $n=1$ internal kink mode [60-63]. In large conventional aspect-ratio tokamaks such as JET, the toroidal rotation driven by the neutral beams is low (typically $v_{\phi}^{J E T}(0) \lesssim 0.1 v_{A}$ ). At such low rotation speeds, the flow effect on the $n=1$ internal kink mode is dominated by the (de)stabilisation of the beam energetic ion population $[64,65]$. In smaller conventional tokamaks, where the fast ion pressure is smaller due to reduced NBI power, but the lower plasma volume results in faster rotation (typically $v_{\phi}^{D I I I-D, A U G}(0) \lesssim 0.2 v_{A}$ ), the rotational effects are a similar order of magnitude as the effects of the fast ions. Indeed, a combination of drift kinetic modelling to assess the change in the potential energy of the mode due to the fast ions, and an MHD stability analysis including toroidal flows, has shown that a competition between these two stabilising mechanisms can occur in ASDEX Upgrade [23] and TEXTOR [22] plasmas, manifest in the experimental behaviour of the sawtooth oscillations. In spherical tokamaks the high beam power per unit volume, low moment of inertia of the plasma and the low toroidal field mean that the toroidal plasma rotation can approach the Alfvén speed. In the presence of such fast toroidal rotation, the stability of the $n=1$ kink mode is often dominated by the strong toroidal flows $[21,26]$. However, since the effect of the energetic ions is highly dependent upon gradients in the fast ion distribution function and local finite orbit width effects $[66,67]$, the fast ion population can be tailored in such a way that the energetic population could still have a significant effect on kink mode stability, even in strongly rotating plasmas. In ITER the toroidal rotation is expected to be small. In an ITER discharge with $\beta_{N}=2.6$, empirical scaling laws have been used to predict that the intrinsic plasma rotation (ie not driven by NBI) could be $V / V_{A}=0.02$ [27]. Predictive transport simulations suggest that toroidal rotation in ITER plasmas with $P_{N B I}=30 \mathrm{MW}$ may be in the range $V / V_{A}=0.05-0.3$ depending on the model employed for the momentum diffusivity [68]. Even if the toroidal rotation can approach a third of the Alfvén speed, it is still likely that the stability of the $n=1$ kink mode will be determined by the fast ion population. The fusion-born $\alpha$ particles have been predicted to be very strongly stabilising in ITER, which is likely to produce long-period sawteeth $[65,69,70]$.

Despite the significant effect of energetic particles, in strongly rotating tokamak plasmas, the effect of toroidal rotation must be considered. This work has emphasised that in order to make a thorough assessment of MHD stability in flowing plasmas, 
consideration must be given to variations in the plasma density and rotation profiles. The stability of the internal kink mode is highly sensitive to these profiles, and small perturbations in either density or toroidal flow can result in a rotationally-stabilised mode being destabilised, and vice versa. Furthermore, the sensitivity of the mode to plasma profiles is also affected by the treatment of the centrifugal effects on the plasma equilibrium. Inconsistent treatment of the equilibrium flow can lead to misleading interpretation of the effect of toroidal rotation for some sets of plasma profiles. In the MAST plasmas analysed here, an inconsistent treatment of the equilibrium flow always leads to an over-estimate of the toroidal rotation required to marginally stabilise the $n=1$ kink mode. Consequently, inconsistent stability analyses typically provide an 'upper bound' for the rotation required to stabilise the mode.

Finally, the extreme sensitivity of the effect of rotation to the plasma profiles has implications for the analysis of experimental data. It is important to consider how variations within the error bars of the measured profiles could impact upon the mode stability. The error on plasma diagnostics is often greater than $10 \%$, whereas a variation of only a few percent can be sufficient to reverse the stabilising/destabilising effect of the toroidal flow. Similarly, the temporal evolution of the plasma profiles may strongly impact on experimental comparisons. The MAST data shown in this paper exemplifies how density profiles can evolve significantly - notably, how the radial gradient of the profile at the $q=1$ surface can change sign - in only a fraction of a confinement time. Thus the stability of the internal kink mode will vary significantly during the evolution of the discharge. Such ephemeral plasma profiles mean that linear modelling including toroidal flows (as presented in this paper, and commonplace in numerical stability analyses) can, at best, give qualitative assessment of experimental mode stability. It is possible that such changes in the plasma profiles could drive the $n=1$ internal kink mode sufficiently unstable to trigger a sawtooth crash, though many other dynamics, most notably the concurrent evolution of the current profile, are involved in the sawtooth phenomenon.

\section{Acknowledgments}

The authors would like to thank Dr E Strumberger, Dr HP Zehrfeld, Dr C Konz and their co-workers at IPP Garching for providing us with the CASTOR-FLOw and Diva codes. This work was partly funded by the United Kingdom Engineering and Physical Sciences Research Council and by the European Communities under the contract of Association between EURATOM and UKAEA, the Fonds National Suisse de la Recherche Scientifique and the Swedish Research Council. The views and opinions expressed herein do not necessarily reflect those of the European Commission.

\section{References}

[1] Ono M et al 2004 Nucl. Fusion 44452 
[2] Counsell G et al 2005 Nucl. Fusion 45 S157

[3] Helander P, Akers RJ and Eriksson L 2005 Phys. Plasmas 12112503

[4] Sontag A et al 2005 Phys. Plasmas 12056112

[5] Gimblett C 1986 Nucl. Fusion 26617

[6] Chu M et al 1995 Phys. Plasmas 22236

[7] Bondeson A and Ward DJ 1994 Phys. Rev. Lett. 722709

[8] Liu YQ, Bondeson A, Gribov Y and Polevoi A 2004 Nucl. Fusion 44232

[9] Garofalo AM et al 2002 Phys. Plasmas 91997

[10] Cooper WA 1988 Plasma Phys. Control. Fusion 301805

[11] Miller R, Waelbroeck F, Hassam A and Waltz R 1995 Phys. Plasmas 23676

[12] Connor JW, Hastie RJ and Taylor JB 2004 Plasma Phys. Control. Fusion 46 B1

[13] Wilson HR 1993 Plasma Phys. Control. Fusion 35885

[14] Furukawa M and Tokuda S 2005 Nucl. Fusion 45377

[15] Saarelma S et al 2007 Plasma Phys. Control. Fusion 4931

[16] Oyama N et al 2005 Nucl. Fusion 45871

[17] Sakamoto Y et al 2004 Plasma Phys. Control. Fusion 46 A299

[18] Burrell K et al 2002 Plasma Phys. Control. Fusion 44 A253

[19] Wahlberg C and Bondeson A 2000 Phys. Plasmas 7923

[20] Waelbroeck FL 1996 Phys. Plasmas 31047

[21] Chapman IT et al 2006 Nucl. Fusion 461009

[22] Chapman IT et al 2008 Nucl. Fusion 48035004

[23] Chapman IT et al 2009 Nucl. Fusion 49035006

[24] Wahlberg C 2005 Plasma Phys. Control. Fusion 47757

[25] Zheng L-J, Chu M and Chen L 1999 Phys. Plasmas 61217

[26] Menard JE et al 2005 Nucl. Fusion 45539

[27] Rice JE et al 2007 Nucl. Fusion 471618

[28] Sabbagh SA et al 2006 Nucl. Fusion 46635

[29] Wilson HR et al 2004 20th IAEA Fusion Energy Conference, Villamoura FT/3-1Ra

[30] Morris AW et al 2005 Fusion Engineering and Design 7567

[31] Peng Y-K M et al 2005 Plasma Phys. Control. Fusion 47 B263

[32] Liu YQ et al 2000 Phys. Plasmas 73681

[33] Snyder PB et al 2007 Nucl. Fusion 47961

[34] Chapman IT et al 2006 Phys. Plasmas 13062511

[35] Aiba N, Tokuda S, Furukawa M, Snyder PB and Chu MS, 2009 accepted Comp. Phys. Comms. 180 PAGE

[36] Kurita G et al. 2003 45th APS Annual Meeting of the Division of Plasma Physics, Albuquerque

[37] Park W et al 2003 Nucl. Fusion 43483

[38] Kleva RG and Guzdar PN 2002 Phys. Plasmas 93013

[39] Wahlberg C, Chapman IT and Graves JP 2009 sub Phys. Plasmas

[40] Guazzotto L, Freidberg JP and Betti R 2008 Phys. Plasmas 15072503

[41] Guazzotto L et al 2004 Phys. Plasmas 11604

[42] Blokland JW et al 2008 J. Comp. Physics 226509

[43] Beliën AJC et al 2002 J. Comp. Physics 18291

[44] Strumberger E et al 2005 Nucl. Fusion 451156

[45] Zehrfeld HP 1999 26th European Physical Society Annual Conference of Plasma Physics, Maastricht, Netherlands Vol 23 Page 1421

[46] Kerner W, Poedts S, Goedbloed JP, Huysmans GTA, Keegan B and Schwarz E 1991 Proc. 18th EPS Conf.on Controlled Fusion and Plasma Physics (Berlin) Geneva: EPS 489

[47] Huysmans GTA, Goedbloed JP and Kerner W 1993 Phys. Fluids B 51545

[48] Bussac M, Edery D, Pellat R and Soule J 1976 Plasma Phys. Controlled Nucl. Fusion Research 1 607 (IAEA, Vienna) 
[49] Graves JP and Wahlberg C 2007 Phys Plasmas 14082504

[50] Wahlberg C 2008 Phys. Rev. Lett. 101115003

[51] Huysmans G, Goedbloed J and Kerner W 1991 Proc. CP90 Conf. on Computational Physics Proc. (Singapore: World Scientific) p371

[52] Ida K et al 1992 Phys. Rev. Lett. 68182

[53] Angioni C, Peeters AG, Pereverzev GV, Ryter F and Tardini G 2003 Phys. Rev. Lett. 90205003

[54] Chapman IT et al 2009 sub Phys Plasmas "The effect of off-axis neutral beam injection on sawtooth stability in ASDEX Upgrade and MAST"

[55] Lao L et al 1990 Nucl. Fusion 301035

[56] Akers RJ et al 2003 Plasma Phys. Control. Fusion 45 A175

[57] Arends ER, Walsh MJ and Lopes Cardozo, NJ 2001 28th European Physical Society Annual Conference of Plasma Physics, Funchal, Portugal Vol 25A Page 589

[58] Strait EJ et al 2007 Phys. Plasmas 14056101

[59] Solomon WM et al 2008 22nd IAEA Fusion Energy Conference, Geneva EX/3-4

[60] Porcelli F 1991 Plasma Phys. Control. Fusion 331601

[61] Betti R and Freidberg JP 1993 Phys. Rev. Lett. 703428

[62] White RB, Rutherford PH, Colestock P and Bussac MN 1988 Phys. Rev. Lett. 602038

[63] Chen L, White RB and Rosenbluth MN 1984 Phys. Rev. Lett. 521122

[64] Chapman IT et al 2007 Phys. Plasmas 14070703

[65] Chapman IT et al 2007 Plasma Phys. Control. Fusion 49 B385

[66] Graves JP 2004 Phys. Rev. Lett. 92185003

[67] Graves JP, Chapman IT, Coda S, Eriksson LG and Johnson T 2009 Phys. Rev. Lett. 102065005

[68] Halpern FD, Kritz AH, Bateman G, Pankin AY, Budny RV and McCune DC 2008 Phys. Plasmas 15062505

[69] Porcelli F, Boucher D and Rosenbluth MN 1996 Plasma Phys. Control. Fusion 382163

[70] Hu B, Betti R and Manickam J 2006 Phys. Plasmas 13112505 


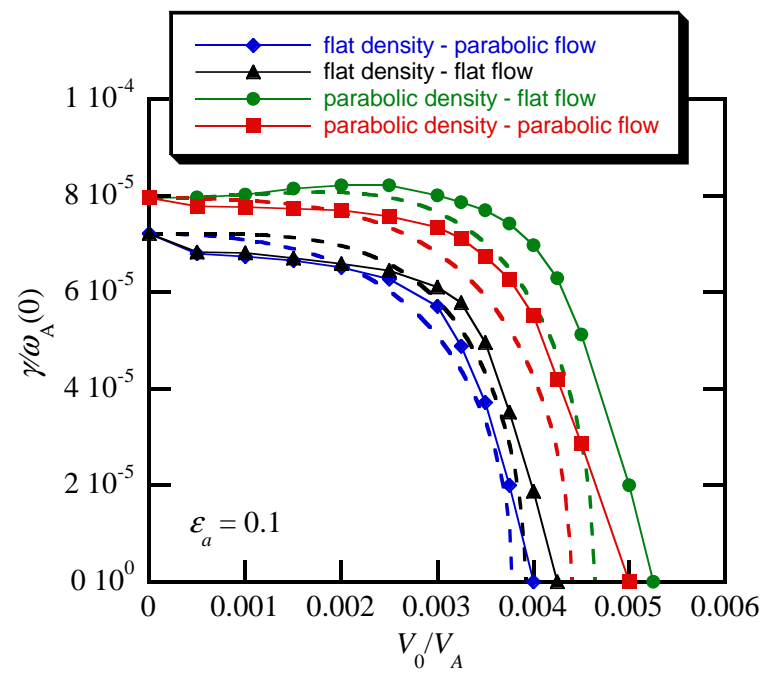

Figure 1. The growth rate of the $n=1$ internal kink mode with respect to the toroidal rotation at the magnetic axis for four combinations of plasma profiles given by equations 13 and 14. The plasma parameters are: $\epsilon_{a}=0.1, \beta_{p}=0.3, \beta_{0}=0.66 \%$, $q_{0}=0.938, r_{1}=0.3 a$. The equilibrium flow is treated consistently. The symbols are from CASTOR-FLOW stability analysis whereas the lines are predicted by analytic theory.

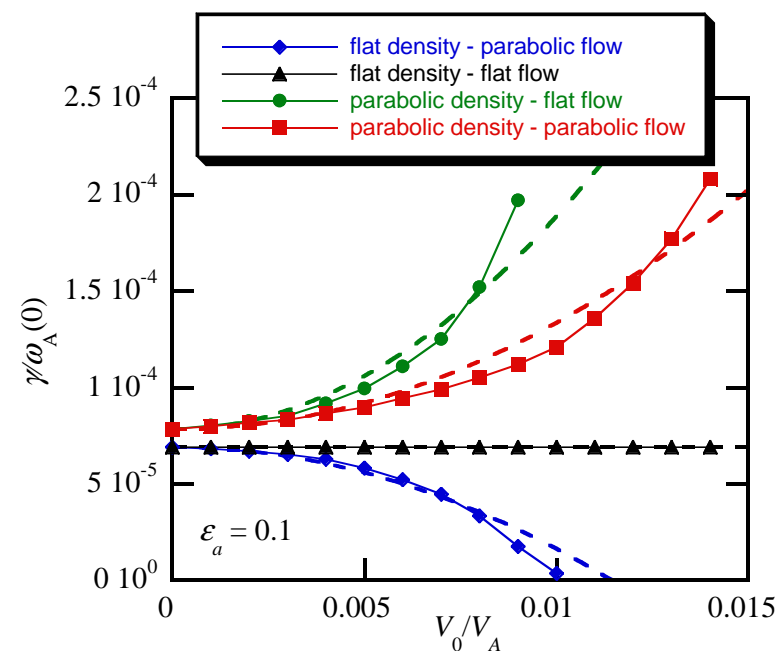

Figure 2. The growth rate of the $n=1$ internal kink mode with respect to the toroidal rotation at the magnetic axis for four combinations of plasma profiles given by equations 13 and 14. The plasma parameters are: $\epsilon_{a}=0.1, \beta_{p}=0.3, \beta_{0}=0.66 \%$, $q_{0}=0.938, r_{1}=0.3 a$. The equilibrium flow is treated inconsistently. The symbols are from MishKA-F stability analysis whereas the lines are predicted by analytic theory. 


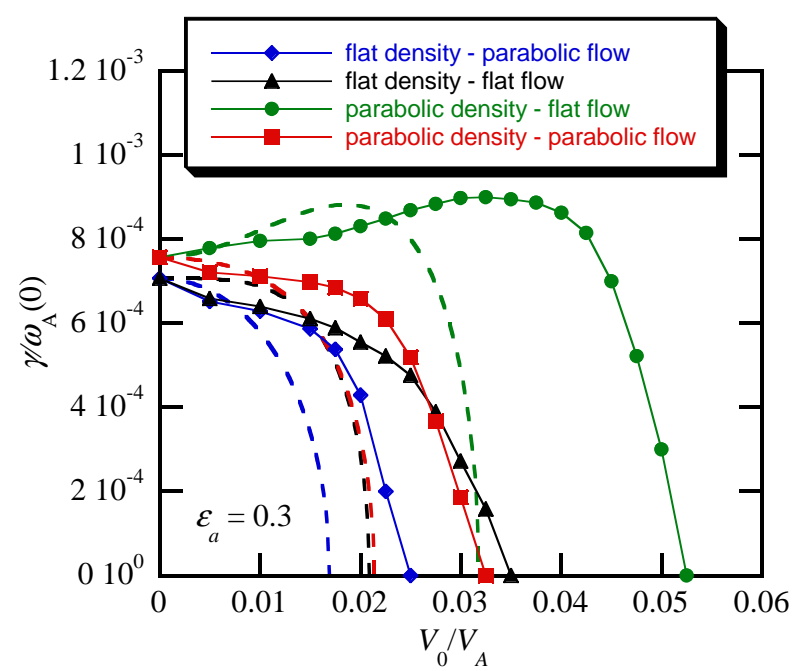

Figure 3. The growth rate of the $n=1$ internal kink mode with respect to the toroidal rotation at the magnetic axis for four combinations of plasma profiles given by equations 13 and 14 . The plasma parameters are: $\epsilon_{a}=0.3, \beta_{p}=0.3, \beta_{0}=5.6 \%$, $q_{0}=0.938, r_{1}=0.3 a$. The equilibrium flow is treated consistently. The symbols are from CASTOR-FLOW stability analysis whereas the lines are predicted by analytic theory.

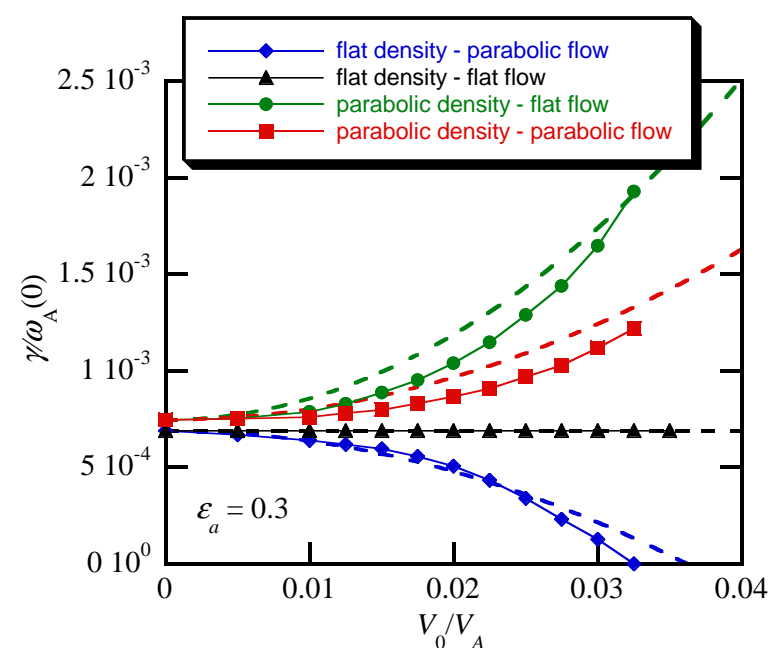

Figure 4. The growth rate of the $n=1$ internal kink mode with respect to the toroidal rotation at the magnetic axis for four combinations of plasma profiles given by equations 13 and 14 . The plasma parameters are: $\epsilon_{a}=0.3, \beta_{p}=0.3, \beta_{0}=5.6 \%$, $q_{0}=0.938, r_{1}=0.3 a$. The equilibrium flow is treated inconsistently. The symbols are from MisHKA-F stability analysis whereas the lines are predicted by analytic theory. 
Figure 5. The growth rate of the $n=1$ internal kink mode with respect to the toroidal rotation at the magnetic axis for four combinations of plasma profiles given by equations 13 and 14 . The plasma parameters are: $\epsilon_{a}=0.3, \beta_{p}=0.3, \beta_{0}=5.6 \%$, $q_{0}=0.75, r_{1}=0.3 a$. The equilibrium flow is treated consistently. The symbols are from CASTOR-FLOW stability analysis whereas the lines are predicted by analytic theory.

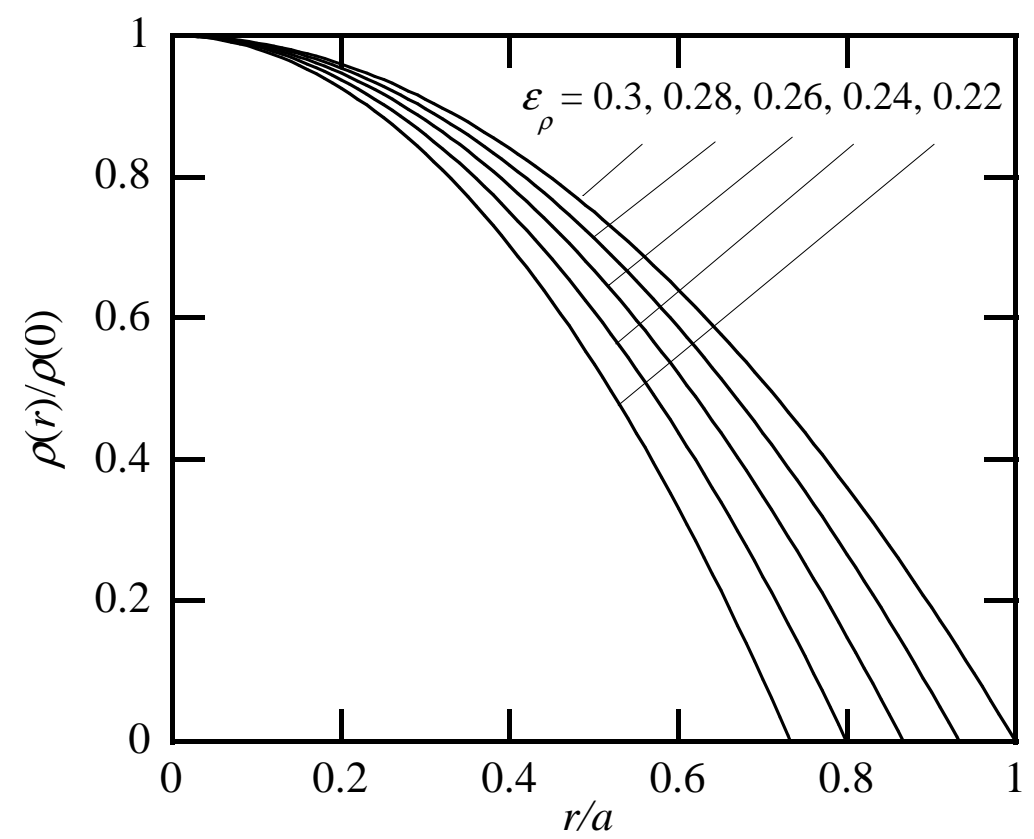

Figure 6. The density profiles given by the parameters in table 1 applied to equation 15 . 


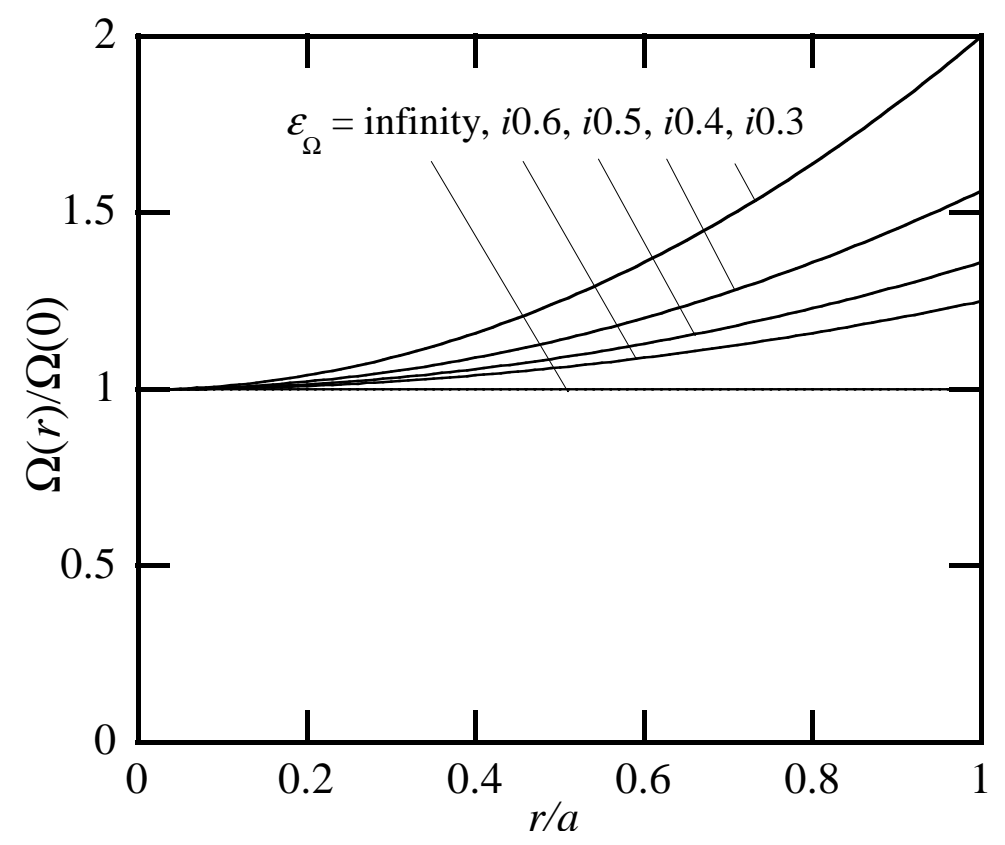

Figure 7. The rotation profiles given by the parameters in table 2 applied to equation 16.

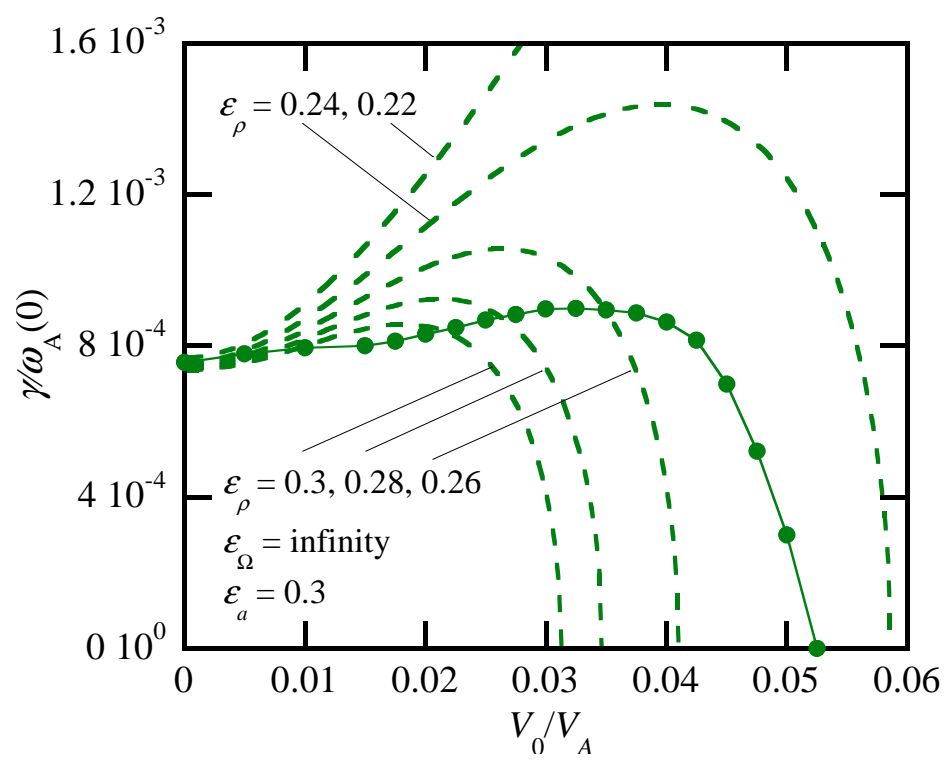

Figure 8. The growth rate of the $n=1$ internal kink mode with respect to the toroidal rotation at the magnetic axis for the different density profiles given by the parameters in table 1 and illustrated in figure 6 . The plasma parameters are: $\epsilon_{a}=0.3$, $\beta_{p}=0.3, \beta_{0}=5.6 \%, q_{0}=0.938, r_{1}=0.3 a$. The solid line represents the numerical calculation for the growth rate with a consistent treatment of the equilibrium flow. 


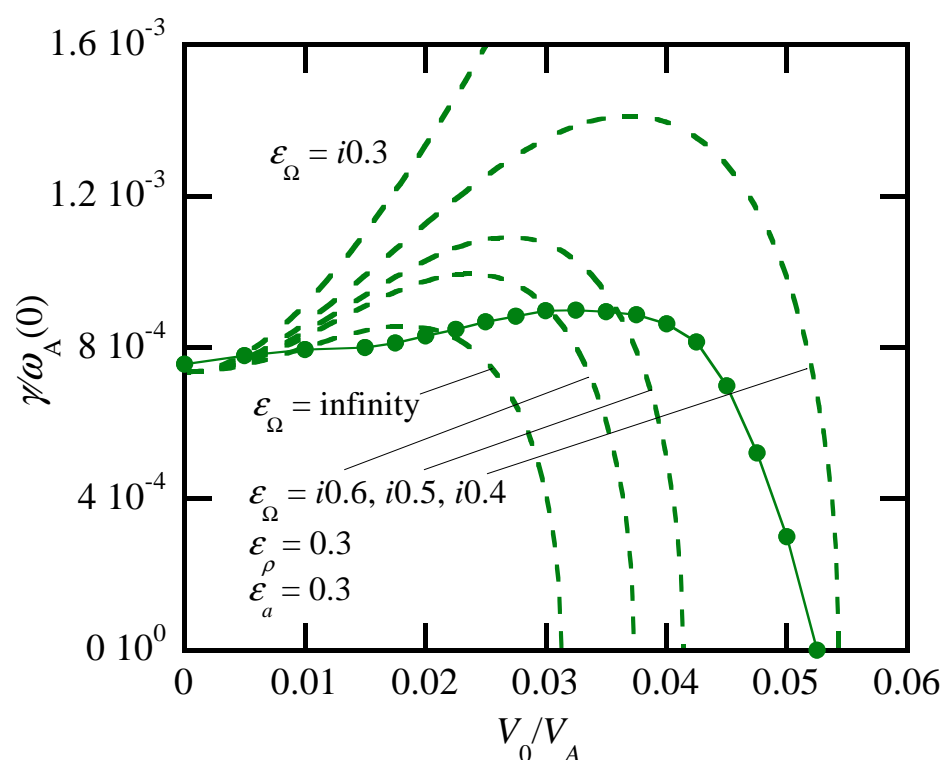

Figure 9. The growth rate of the $n=1$ internal kink mode with respect to the toroidal rotation at the magnetic axis for the different rotation profiles given by the parameters in table 2 and illustrated in figure 7. The plasma parameters are: $\epsilon_{a}=0.3$, $\beta_{p}=0.3, \beta_{0}=5.6 \%, q_{0}=0.938, r_{1}=0.3 a$. The solid line represents the numerical calculation for the growth rate with a consistent treatment of the equilibrium flow.

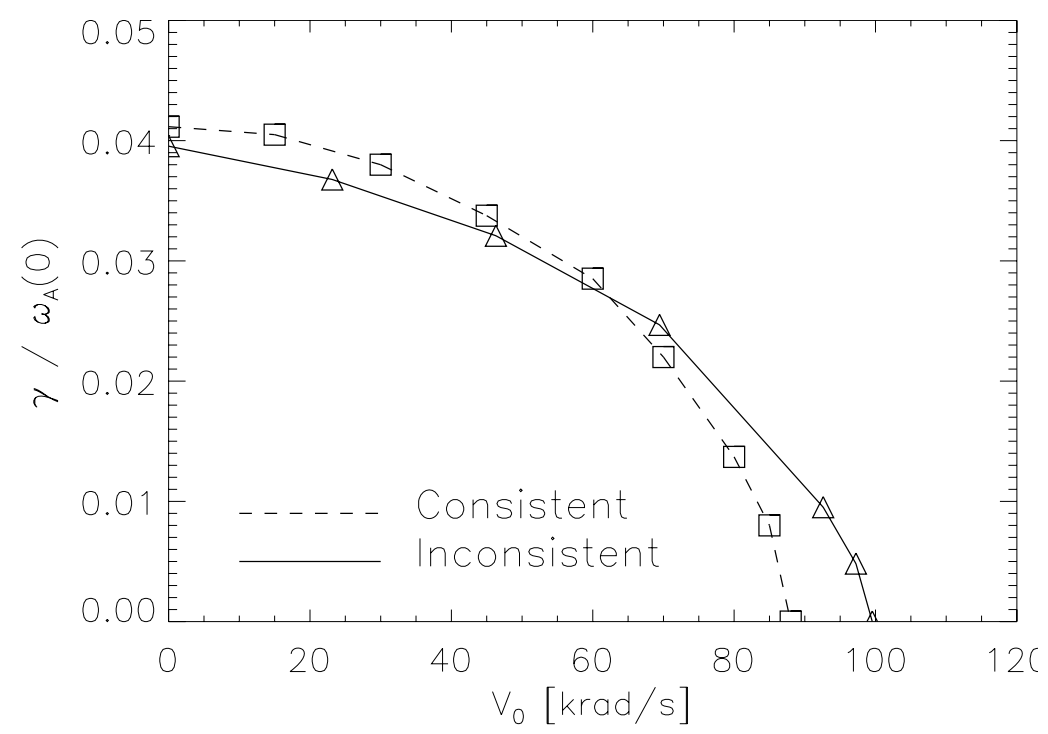

Figure 10. The growth rate of the $n=1$ internal kink mode with respect to the toroidal rotation velocity at the $q=1$ surface for sawtoothing MAST discharge 13035 [21] analysed with a self-consistent rotating equilibrium and an inconsistent static equilibrium. 


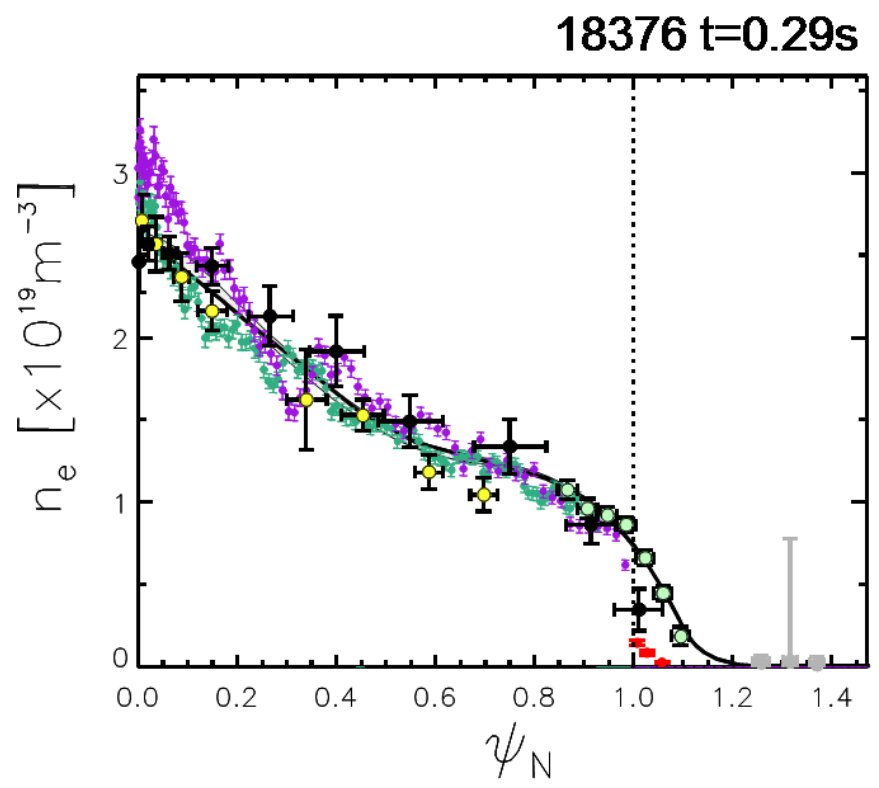

Figure 11. The density profile for MAST discharge 18376.

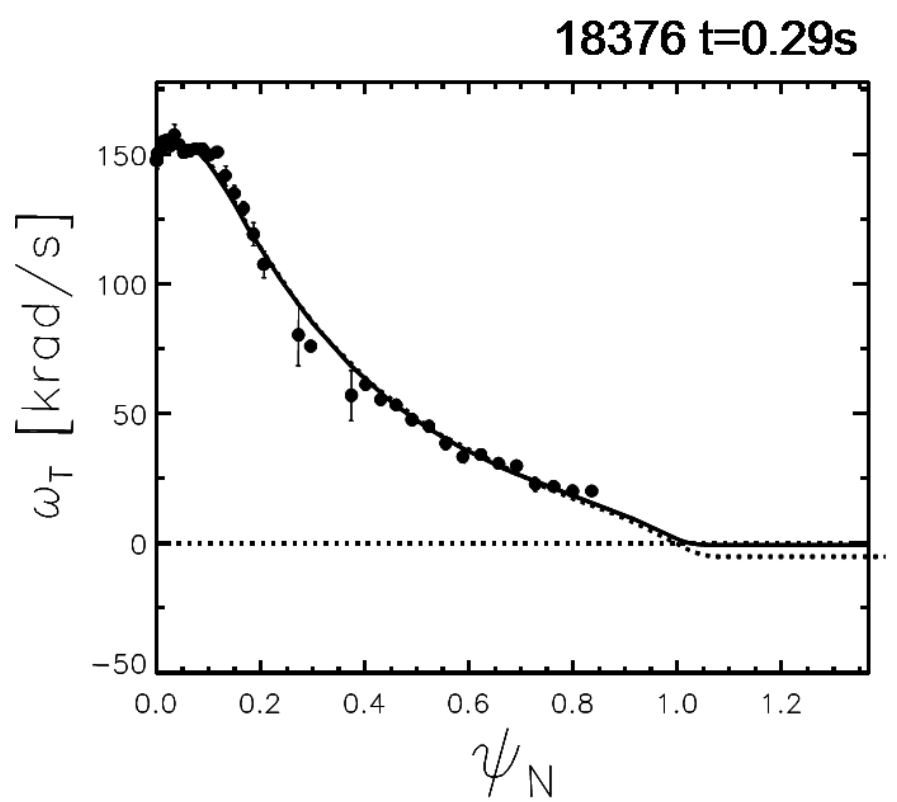

Figure 12. The rotation profile for MAST discharge 18376. 


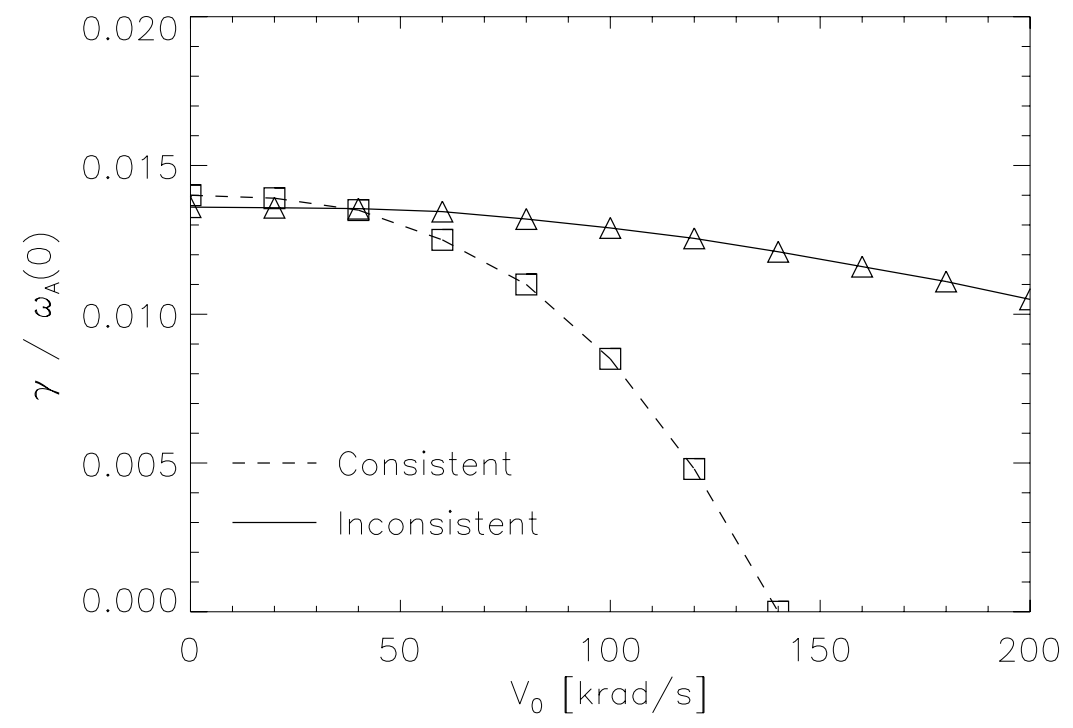

Figure 13. The growth rate of the $n=1$ internal kink mode with respect to the toroidal rotation velocity at the $q=1$ surface for sawtoothing MAST discharge 18376 analysed with a self-consistent rotating equilibrium and an inconsistent static equilibrium.

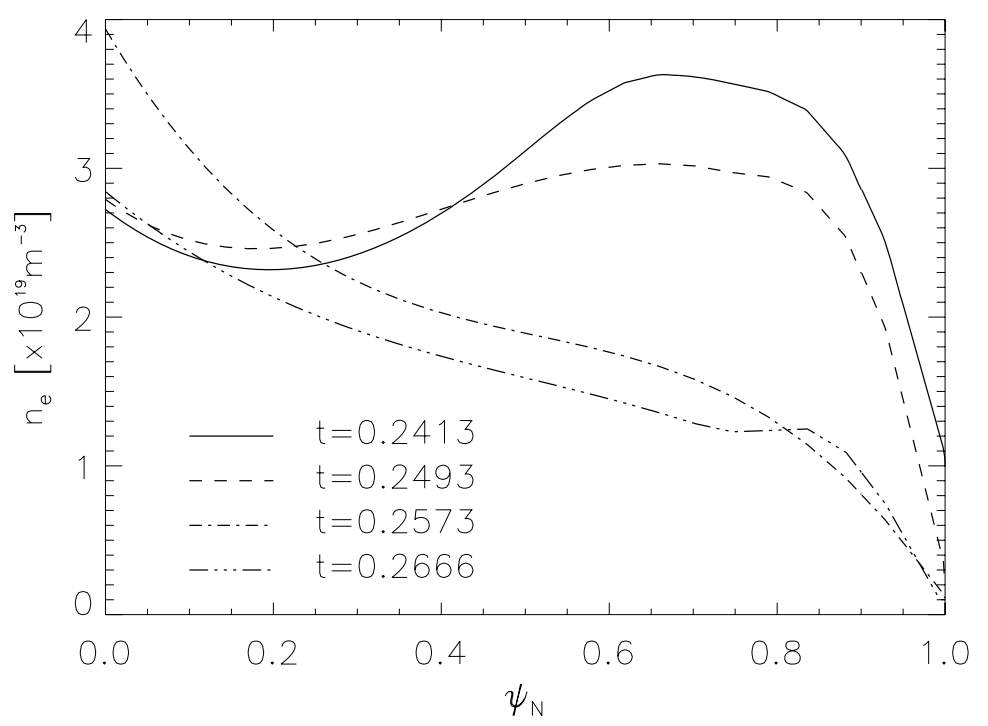

Figure 14. The density profile in MAST shot 21430 at various times during the discharge evolution. 


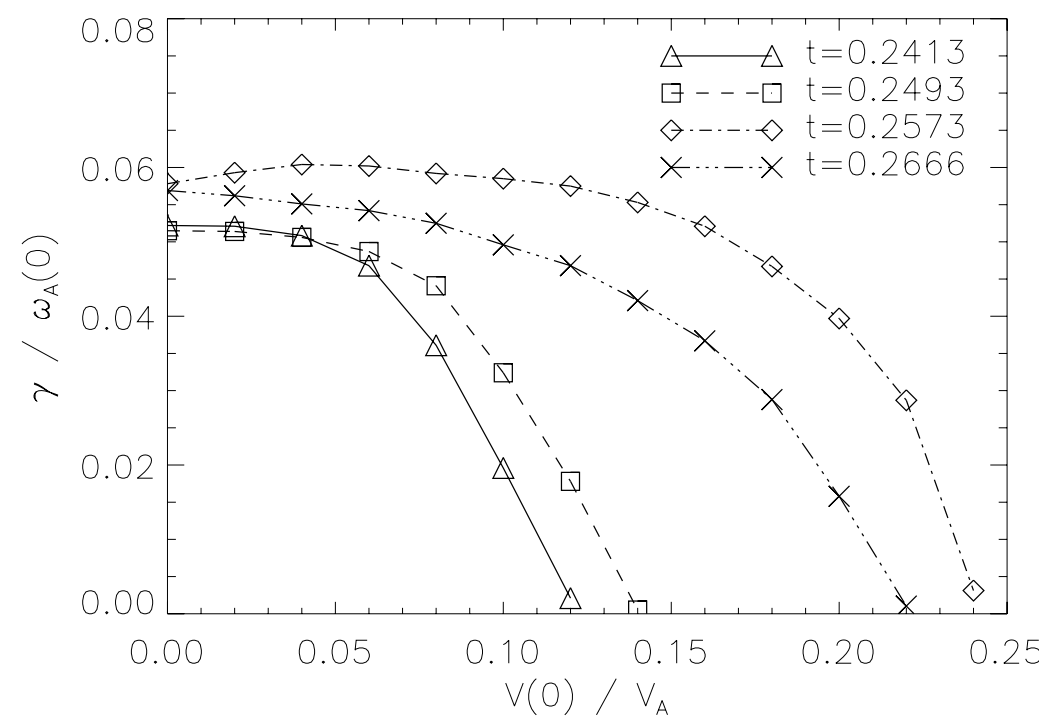

Figure 15. The growth rate of the $n=1$ internal kink mode calculated using the consistent treatment of the equilibrium flow for the different density profiles during the evolution of MAST shot 21430, as illustrated in figure 14 .

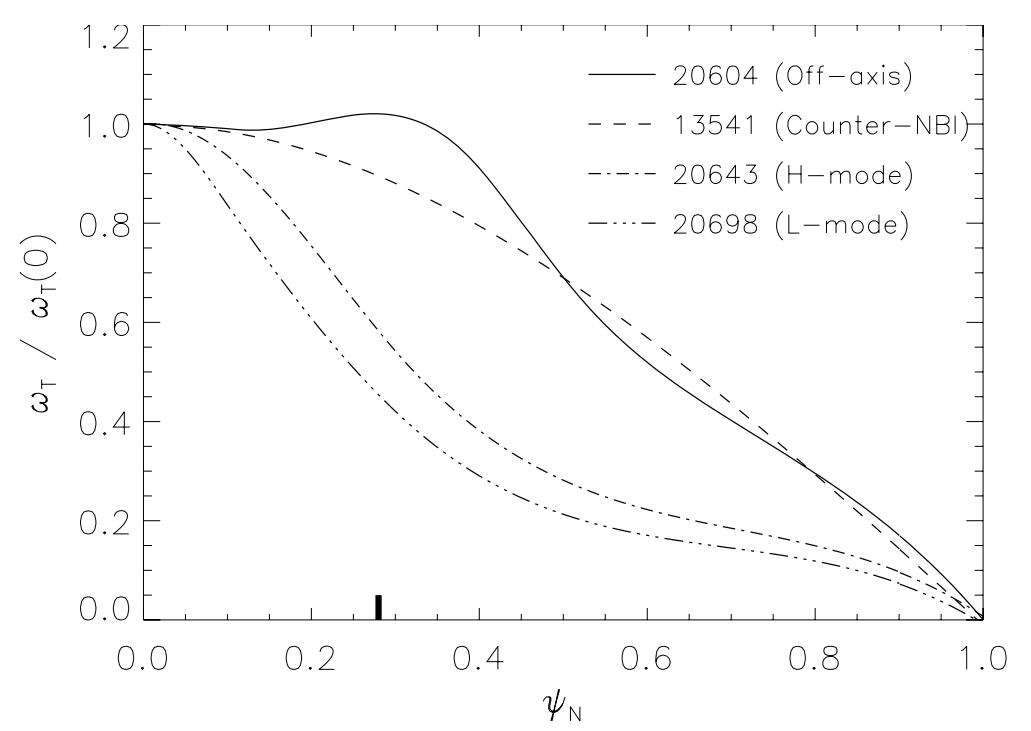

Figure 16. The rotation profile in typical MAST discharges with off-axis neutral beam injection (20604), counter-NBI (13541), on=-axis NBI in H-mode (20643) and L-mode (20698). The position of the $q=1$ surface in shot 20643 (as used in the stability analysis shown in figure 17) is given by the tick mark. 


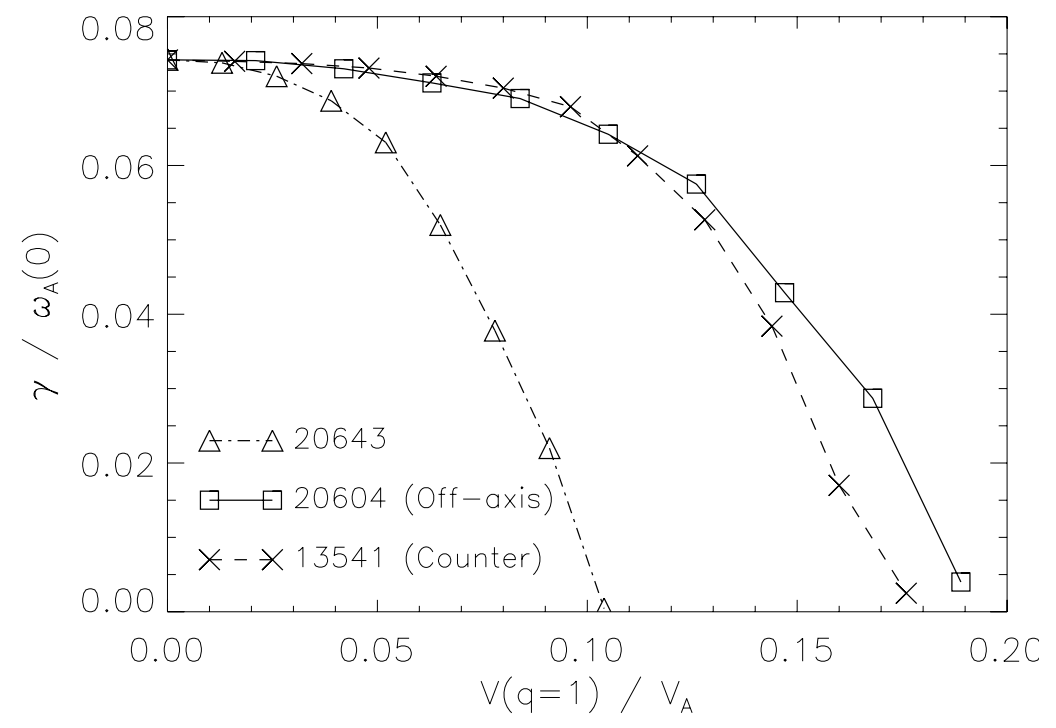

Figure 17. The growth rate of the $n=1$ internal kink mode in MAST discharge 20643 calculated using the consistent treatment of the equilibrium flow for the different rotation profiles illustrated in figure 16 . 